\title{
Treatment Patterns and Lipid Profile in Patients with Familial Hypercholesterolemia in Japan
}

\author{
Tamio Teramoto ${ }^{1}$, Takahito $\mathrm{Kai}^{2}$, Asuka Ozaki ${ }^{2}$, Bruce Crawford ${ }^{3}$, Hidenori Arai $^{4}$ and Shizuya Yamashita ${ }^{5,6}$ \\ ${ }^{1}$ Teikyo Academic Research Center, Tokyo, Japan \\ ${ }^{2}$ Diabetes \& Cardiovascular Medical Operations, Sanofi, Tokyo, Japan \\ ${ }^{3}$ Real World Evidence \& Insights, inVentiv Health, Tokyo, Japan \\ ${ }^{4}$ National Center for Geriatrics and Gerontology, Aichi, Japan \\ ${ }^{5}$ Rinku General Medical Center, Osaka, Japan \\ ${ }^{6}$ Department of Community Medicine \& Department of Cardiovascular Medicine, Osaka University Graduate School of Medicine, \\ Osaka, Japan
}

\begin{abstract}
Aim: To evaluate the epidemiology and real-world treatment patterns associated with lipid-modifying therapies (LMTs) among groups of Japanese patients with familial hypercholesterolemia (FH).

Methods: A retrospective observational study was conducted using an electronic hospital-based administrative claims database and electronic medical records. Patients with existing diagnosis of $\mathrm{FH}(\mathrm{FH}-$ D) and patients with suspected FH (FH-S) defined by low-density lipoprotein cholesterol (LDL-C) $\geq 190 \mathrm{mg} / \mathrm{dL}$ were included, and medical records of hospitals across Japan were analyzed to assess the diagnostic status, management of LDL-C levels, and treatment patterns.

Results: Among the 3,495 patients who met the inclusion criteria, 193 patients were FH-D and 3,339 patients were FH-S. Among them, $83.5 \%$ had not achieved the LDL-C of $<100 \mathrm{mg} / \mathrm{dL}$ recommended for patients with FH at the index date. Mean LDL-C levels for all patients and for FH-D and FH-S patients were $145.8 \mathrm{mg} / \mathrm{dL}, 119.2 \mathrm{mg} / \mathrm{dL}$, and $147.6 \mathrm{mg} / \mathrm{dL}$, respectively. $44.5 \%$ of the patients were not currently treated with LMTs. High-intensity statins were used only in $19.2 \%$ and $2.3 \%$ of the FH-D and FH-S patients, respectively. Furthermore, among the FH-D and FH-S statintreated patients, $61(69.3 \%)$ and $1,059(89.7 \%)$ remained on monotherapy even when their LDL-C was $\geq 100 \mathrm{mg} / \mathrm{dL}$.

Conclusions: Treatment and management of LDL-C in Japanese FH patients remain suboptimal. The results suggest that $\mathrm{FH}$ is underdiagnosed in real-world, routine clinical practice in Japan. There is an urgent need to improve the diagnostic rate of $\mathrm{FH}$ and to provide the appropriate therapy to achieve the recommended LDL-C levels of $<100 \mathrm{mg} / \mathrm{dL}$ or a more than $50 \%$ reduction for patients with FH in Japan.
\end{abstract}

\section{See editorial vol. 25: 568-569}

Key words: Familial hypercholesterolemia, LDL-C, Database analysis, Treatment patterns

Copyright@2018 Japan Atherosclerosis Society

This article is distributed under the terms of the latest version of CC BY-NC-SA defined by the Creative Commons Attribution License.

\section{Introduction}

Familial hypercholesterolemia $(\mathrm{FH})$ is a common

Address for correspondence: Tamio Teramoto, Teikyo Academic

Research Center, 2-11-1, Kaga, Itabashi-ku, Tokyo 173-8605 Japan

E-mail: ttera@med.teikyo-u.ac.jp

Received: June 28, 2017

Accepted for publication: November 23, 2017 autosomal genetic disorder characterized by the triad of high low-density lipoprotein cholesterol (LDL-C), premature coronary artery disease (CAD), and tendon/ cutaneous xanthoma ${ }^{1-3)}$. Historically, the frequency of clinical homozygous $\mathrm{FH}(\mathrm{HoFH})$ has been estimated at 1 in a million, and for heterozygous $\mathrm{FH}(\mathrm{HeFH}), 1$ in 500 throughout the world ${ }^{4)}$. A recent report has also demonstrated a higher prevalence of $\mathrm{HoFH}$ and $\mathrm{HeFH}$ among the general population in Japan, estimated to 
be $1 / 171,167$ and $1 / 208$, respectively ${ }^{5)}$. It is estimated that there are more than 30 million patients with $\mathrm{FH}$ worldwide ${ }^{6,7)}$, including at least $300,000 \mathrm{HeFH}$ patients in Japan ${ }^{1)}$. Patients with FH have excessive high LDL-C levels due to pathological genetic mutations and are at a significantly higher risk for cardiovascular diseases $^{8-11)}$. The Japanese Atherosclerosis Society (JAS) guidelines have reported that patients with $\mathrm{HeFH}$ develop CAD typically before reaching the age of 55 in men and of 65 in women ${ }^{1)}$, and a Japanese cohort study found that $70 \%$ of $\mathrm{HeFH}$ patients died of $\mathrm{CAD}^{12)}$. When FH patients are left untreated, the risk for developing CAD is 20 times greater than that of $\mathrm{FH}$ patients treated with cholesterol-lowering medications ${ }^{13}$.

While early diagnosis and treatment for patients with $\mathrm{FH}$ are recommended to prevent $\mathrm{CAD}^{1,14)}$, most individuals with $\mathrm{FH}$ remain undiagnosed and untreated worldwide. The European Atherosclerosis Society (EAS) published a Consensus Statement in 2013 warning of the severe underdiagnosis of $\mathrm{FH}$ and its negative consequences ${ }^{15)}$. The vast majority of the diagnosed $\mathrm{FH}$ is categorized as $\mathrm{HeFH}$, and Nordestgaard et al. estimated that less than $1 \%$ of $\mathrm{HeFH}$ patients were diagnosed in most countries ${ }^{16)}$. Among those diagnosed and treated, most were still unable to achieve the recommended LDL-C level targets, resulting in up to a 13 -fold increased risk of $\mathrm{CAD}^{16)}$. It is speculated that Japan is no exception in this regard ${ }^{16)}$.

The 2012 and 2017 JAS guidelines and 2016 European Society of Cardiology (ESC)/EAS guidelines recommend a target of LDL-C $<100 \mathrm{mg} / \mathrm{dL}$ in patients with $\mathrm{FH}$ without atherosclerotic cardiovascular disease $(A S C V D)^{1,2,15)}$. Given that this recommendation is known to be difficult for $\mathrm{FH}$ patients to achieve in routine clinical practice, reduction of LDL-C by more than $50 \%$ is set as the secondary management target ${ }^{1)}$. The consensus is that lifestyle modification alone is generally not sufficient to achieve normalization of the lipid profile in $\mathrm{FH}$ patients; therefore, drug therapy is required, with statins recommended as the first line drug therapy ${ }^{1,13)}$. Previous clinical trials have shown that FH can be effectively treated with lipid-modifying therapies (LMTs) such as statins by reducing LDL-C levels and the risk of cardiovascular events ${ }^{17},{ }^{18}$. For patients in whom target LDL-C levels cannot be achieved using conventional statin therapy, more intensive therapies using other LMTs in combination with a statin are recommended ${ }^{1)}$.

Currently, the proportion of patients diagnosed with $\mathrm{FH}$ and treatment patterns for FH with LMTs in real-world clinical settings in Japan are unknown. Two previous studies have reported the prevalence of $\mathrm{FH}$ patients, including those treated with LMTs. Teramoto et al. (2005) investigated patients who received LMTs for more than 3 months in an outpatient setting in Japan and reported that $3.4 \%$ of these patients had received a diagnosis of $\mathrm{FH}^{19}$. A more recent study reported that $0.6 \%$ of the statin-treated patients who had not achieved the recommended LDL-C targets had a diagnosis of $\mathrm{FH}^{20)}$. With the widespread use of statins and non-statin LMTs for LDL-C lowering in recent years, diagnosis of $\mathrm{FH}$ has become more challenging ${ }^{20)}$. Given the high risk of CAD and the excessive mortality risk among undiagnosed and undertreated $\mathrm{FH}$ patients, there is an urgent need to better understand the management of LDL-C levels for patients with FH in Japan.

\section{Aim}

The objective of the current study was to conduct a cross-sectional analysis to evaluate the epidemiology and real-world treatment patterns associated with LMTs, and to understand the current LDL-C levels among Japanese patients with diagnosed FH (FH-D) or suspected FH (FH-S).

\section{Methods}

\section{Database}

This was a retrospective observational study. The Medical Data Vision database, an electronic hospitalbased administrative claims database, was used for this study. The database consists of inpatient and outpatient medical care data from hospitals across Japan. All hospitals in the database were for acute-care, with an average bed number of 350 . The database contains anonymized patient-level information on demographics, clinical diagnoses, procedures, prescriptions, and laboratory tests.

\section{Patient Selection}

Patients meeting the following inclusion criteria were selected for inclusion in the study (Fig. 1): at least one recorded LDL-C value (measured by direct assay) in 2013, with the most recent LDL-C measurement in 2013 defined at the index date; evidence of either a diagnosis of $\mathrm{FH}$ and/or LDL-C $\geq 190 \mathrm{mg} / \mathrm{dL}$ during the 2-year period prior to the index date; and $\geq 20$ years of age at the index date. In order to ensure that the complete medical history was covered and to determine prior LMT use, the included patients were also required to have $\geq 2$ years of continuous representation in the database prior to the index date. Patients who did not have an LDL-C level of $\geq 190 \mathrm{mg} / \mathrm{dL}$ during the 2-year pre-index period were not included in the current analysis unless they had a diagnosis of $\mathrm{FH}$. 


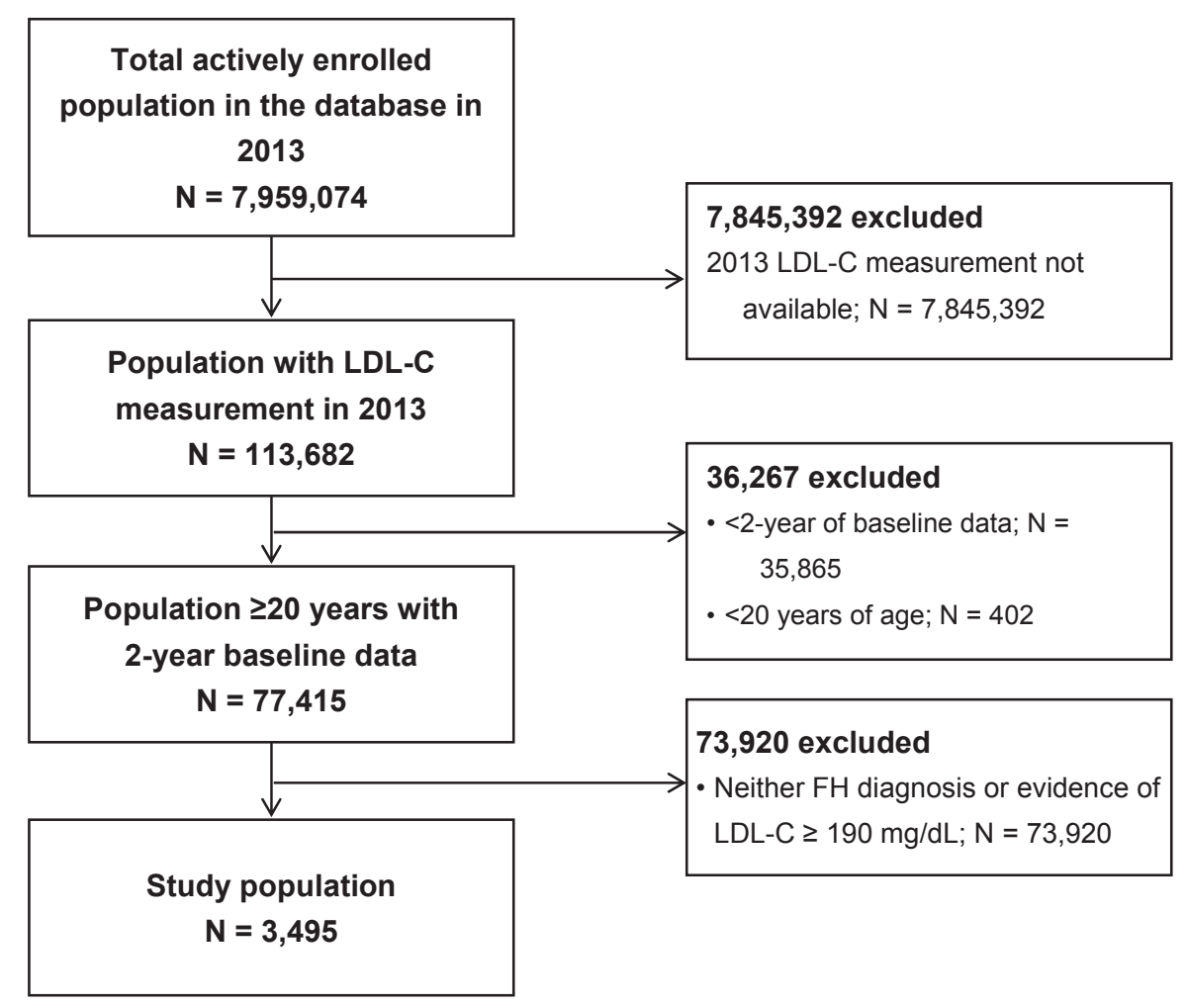

Fig. 1. Patient selection

Given that FH is likely to be underdiagnosed in Japan, our study included the following primary and secondary populations: FH-D and FH-S patients, respectively. FH-D patients were defined as patients with at least one record of FH diagnosis entered by attending physicians during the 2-year period prior to the index date, and FH-S patients were defined as patients with at least one record of LDL-C level $\geq 190 \mathrm{mg} / \mathrm{dL}$ during the 2-year period prior to the index date.

\section{Determination of Treatment Patterns}

Based on the medication utilization status at the index date, patients were classified into the following three categories: "current LMT therapy" if a recorded LMT prescription was present on the index date or within 30 days prior to it (scenario A or B in Fig. 2); "previous LMT therapy" if not currently treated but evidence of a prior recorded LMT during the 2-year pre-index period was present (scenario C in Fig. 2); and "no LMT therapy" if no recorded LMT was present during the 2-year pre-index period.

Current LMT therapy was further classified into three mutually exclusive categories: high-intensity statin therapy with or without other non-statin LMT; lowto-moderate-intensity statin with or without other nonstatin LMT; and non-statin LMT only. High-intensity statin therapy was defined as atorvastatin $\geq 20 \mathrm{mg} /$ day, rosuvastatin $\geq 10 \mathrm{mg} /$ day, and pitavastatin $4 \mathrm{mg} /$ day. All other statins and doses were defined as low-tomoderate-intensity statins in the present study. A third category was used to categorize non-statin LMT only without statin use. This classification scheme is different from the statin classification according to the 2013 American College of Cardiology (ACC)/American Heart Association (AHA) guidelines, which consider highintensity statins to consist of atorvastatin $\geq 40 \mathrm{mg}$ or rosuvastatin $\geq 20 \mathrm{mg}^{21)}$. Non-statin LMTs included ezetimibe, niacin (nicotinic acid), and bile acid sequestrants (cholestyramine, colestimide).

\section{Statistical Analyses}

All statistical analyses in this study were descriptive. Baseline characteristics, LMT utilization, and LDL-C levels were summarized using proportions and mean \pm standard deviation (SD) as appropriate. All analyses were conducted with SAS software version 9.4.

\section{Results}

\section{Patient Characteristics}

A total of 3,495 patients met the inclusion criteria. Table 1 presents the baseline characteristics of all patients $(N=3,495)$, FH-D patients $(N=193)$, and FH-S patients $(N=3,339$, including 37 overlapping with 


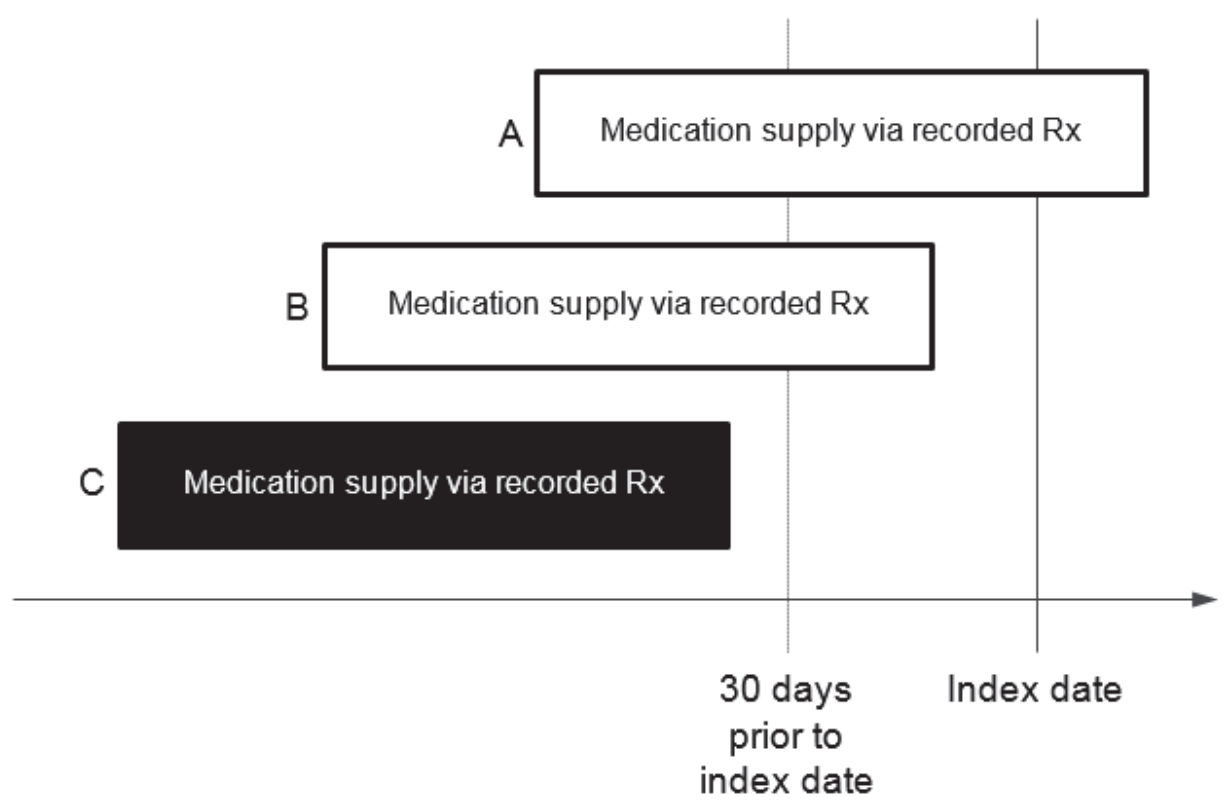

\begin{abstract}
Both white bars representing scenarios A and B (medication supply via recorded prescription on or within 30 days prior to the index date) define the patient as being treated as of index date. The black bar representing scenario $\mathrm{C}$ (medication supply via recorded prescription before 30 days prior to the index date) defines the patient as not being treated as of index date.
\end{abstract}

Fig. 2. Determination of treatment status at index date

FH-D) at the index date. The average ages (SD) for all patients, FH-D patients, and FH-S patients were 63 (13.9), 67 (13.3), and 62 (13.9) years, respectively. The proportion of males for all patients, FH-D patients, and $\mathrm{FH}-\mathrm{S}$ patients were $40.3 \%, 42.0 \%$, and $40.1 \%$, respectively. All diagnosed $\mathrm{FH}$ patients were classified as $\mathrm{HeFH}$; none of the patients had $\mathrm{HoFH}$.

\section{Diagnosis of FH}

Among all 3,495 patients, 5.5\% $(N=193)$ had a diagnosis of $\mathrm{FH}$. When focused on only the 1,708 and 103 patients who were currently receiving any statins and high-intensity statin, the rate of diagnosis of $\mathrm{FH}$ increased to $8.3 \%(N=141)$ and $35.9 \%(N=37)$, respectively.

\section{LDL-C Management}

Fig. 3 details the LDL-C levels at the index date for all patients, FH-D patients, and FH-S patients. The mean LDL-C levels for all patients, FH-D patients, and FH-S patients were $145.8 \mathrm{mg} / \mathrm{dL}, 119.2 \mathrm{mg} / \mathrm{dL}$, and $147.6 \mathrm{mg} / \mathrm{dL}$, which indicates a $16.5 \%, 32.1 \%$, and $15.6 \%$ achievement rate of the recommended LDL-C levels of $<100 \mathrm{mg} / \mathrm{dL}$, respectively. Moreover, $42.7 \%$
$(N=35)$ of the FH-D patients with CAD, 24.3\% ( $N=$ $27)$ of the FH-D patients without CAD, $23.9 \%(N=$ 123) of the FH-S patients with CAD, and $14.1 \%(N=$ 397) of the FH-S patients without CAD had achieved the LDL-C levels of $<100 \mathrm{mg} / \mathrm{dL}$. In the FH-D and FH-S patients with CAD, $13.4 \%(N=11)$ and $3.7 \%$ $(N=19)$ had LDL-C levels of $<70 \mathrm{mg} / \mathrm{dL}$, respectively.

The achieved LDL-C levels were also dependent on the current treatment type. In FH-D and FH-S patients treated with statins, approximately $37.0 \%(N=57)$ and $24.0 \%(N=447)$, respectively, achieved an LDL-C of $<100 \mathrm{mg} / \mathrm{dL}$, a greater proportion than that achieved by patients treated with non-statin LMT $(21.1 \%$ for FH-D and $5.5 \%$ for FH-S) or not treated with LMT at all (5.0\% for FH-D and $4.8 \%$ for FH-S).

\section{Utilization of LMT}

LMT utilization for all patients, FH-D patients, and FH-S patients is presented in Fig. 4. For all patients, nearly half (44.5\%) were currently not treated with any LMT, of which $35.3 \%$ received no LMT therapy over the 2-year period prior to the index date, and the remaining patients $(9.2 \%)$ had previous LMT therapy prior to the index date. Among patients on current 
Table 1. Baseline characteristics of all patients, FH-D patients, and FH-S patients

\begin{tabular}{|c|c|c|c|}
\hline Characteristics & $\begin{array}{l}\text { All patients } \\
(N=3,495)\end{array}$ & $\begin{array}{l}\text { FH-D patients } \\
\qquad(N=193)\end{array}$ & $\begin{array}{l}\text { FH-S patients } \\
\quad(N=3,339)\end{array}$ \\
\hline \multicolumn{4}{|l|}{ Age } \\
\hline Mean (SD), years & $62.7(13.9)$ & $67.2(13.3)$ & $62.4(13.9)$ \\
\hline \multicolumn{4}{|l|}{ Sex } \\
\hline Male, n (\%) & $1,409(40.3)$ & $81(42.0)$ & $1,340(40.1)$ \\
\hline \multicolumn{4}{|l|}{ Living location } \\
\hline Metropolitan, n (\%) & $2,843(81.3)$ & $161(83.4)$ & $2,710(81.2)$ \\
\hline \multicolumn{4}{|c|}{ Comorbid conditions, $\mathrm{n}(\%)$} \\
\hline CAD & $584(16.7)$ & $82(42.5)$ & $515(15.4)$ \\
\hline Ischemic stroke & $42(1.2)$ & $2(1.0)$ & $41(1.2)$ \\
\hline PAD & $239(6.8)$ & $24(12.4)$ & $221(6.6)$ \\
\hline Diabetes & $1,001(28.6)$ & $74(38.3)$ & $937(28.1)$ \\
\hline CKD Stage IV - V ${ }^{1}$ & $424(12.1)$ & $25(13.0)$ & $402(12.0)$ \\
\hline Hypertension & $1,934(55.3)$ & $133(68.9)$ & $1,818(54.4 \%)$ \\
\hline
\end{tabular}

CAD: Coronary artery disease, PAD: Peripheral artery disease, CKD: Chronic kidney disease, FH: Familial hypercholesterolemia, FH-D: Diagnosed FH patients, FH-S: Suspected FH patients, SD: Standard deviation

${ }^{1} \mathrm{CKD}$ Stage IV-V: $30>$ eGFR or dialysis

LMT therapy $(55.5 \%$ of all patients), low-to-moderate-intensity statins were dominantly used (45.9\%). In comparison, high-intensity statins and non-statin LMTs only were used by $2.9 \%$ and $6.6 \%$ of the patients, respectively.

For FH-D patients, $81.9 \%$ were on current LMT therapy. The majority of the FH-D patients (53.9\%) were on low-to-moderate-intensity statins, and 19.2\% and $8.8 \%$ of the FH-D patients were on high-intensity statins and non-statin LMTs, respectively.

For the FH-S patients, $54.1 \%$ were on current LMT therapy, with the majority $(45.3 \%)$ receiving lowto-moderate-intensity statins. Among the FH-S patients, $2.3 \%$ and $6.5 \%$ were on high-intensity statins and non-statin LMTs, respectively.

A detailed breakdown of the therapies according to the LDL-C range for all patients, FH-D patients, and FH-S patients is presented in the Supplementary Table. Among the FH-D and FH-S patients with LDL-C $\geq 100 \mathrm{mg} / \mathrm{dL}$ on any statin treatment, we found that $69.3 \%$ and $89.7 \%$ remained on monotherapy, respectively. Patients with FH-D were more likely to be treated with high-intensity statins (19.2\%) compared with FH-S patients (2.3\%).

\section{Discussion}

This study investigated real-world treatment patterns and clinical characteristics of LMTs among Japanese patients with FH-D or FH-S using a large hospital administrative claims database. To our knowledge, this is the first study that specifically focused on real- world clinical practice associated with FH in Japan.

$\mathrm{FH}$ is a common genetic cause of premature $\mathrm{CAD}$ due to lifelong elevated LDL-C levels. With the right diagnosis, patients with $\mathrm{FH}$ can be treated with LDLC-lowering medication to attenuate the development of atherosclerosis and to prevent $\mathrm{CAD}^{16)}$. Current ESC/ EAS guidelines recommend treatment to be initiated as early as possible to achieve the target LDL-C levels of $<70$ and $<100 \mathrm{mg} / \mathrm{dL}$ in $\mathrm{FH}$ patients with and without ASCVD, respectively. This is also reflected in the newly revised 2017 JAS guidelines, which recommend target LDL-C levels of $<70$ and $<100 \mathrm{mg} / \mathrm{dL}$ in $\mathrm{FH}$ patients with and without $\mathrm{CAD}$, respectively ${ }^{1,2,15)}$.

The average LDL-C levels among FH patients in the current study (i.e., $145.8 \mathrm{mg} / \mathrm{dL}$ for all patients and $119.2 \mathrm{mg} / \mathrm{dL}$ for FH-D patients who were $\mathrm{HeFH}$ with or without LMT) were considerably lower than those previously reported in a study based on nationwide surveillance data from 1996-1998 (i.e., $248 \mathrm{mg} /$ $\mathrm{dL}$ for $\mathrm{HeFH}$ patients without LMT) ${ }^{22)}$. The widespread use of statins and other LMTs for hypercholesterolemia as well as the rising $\mathrm{FH}$ awareness among physicians may have improved LDL-C management over the last 20 years. However, the findings of this study suggest that LDL-C levels are still not adequately managed in the majority of the Japanese patients with either FH-D or FH-S, as $67.9 \%(N=131)$ and $84.4 \%$ $(N=2,819)$ of the FH-D and FH-S patients, respectively, had LDL-C levels of $\geq 100 \mathrm{mg} / \mathrm{dL}$, which exceed the LDL-C target level recommended by the JAS guidelines. Although slightly higher achievements of LDL-C levels of $<100 \mathrm{mg} / \mathrm{dL}$ were observed in the FH-D 


\section{A) Total Patients}

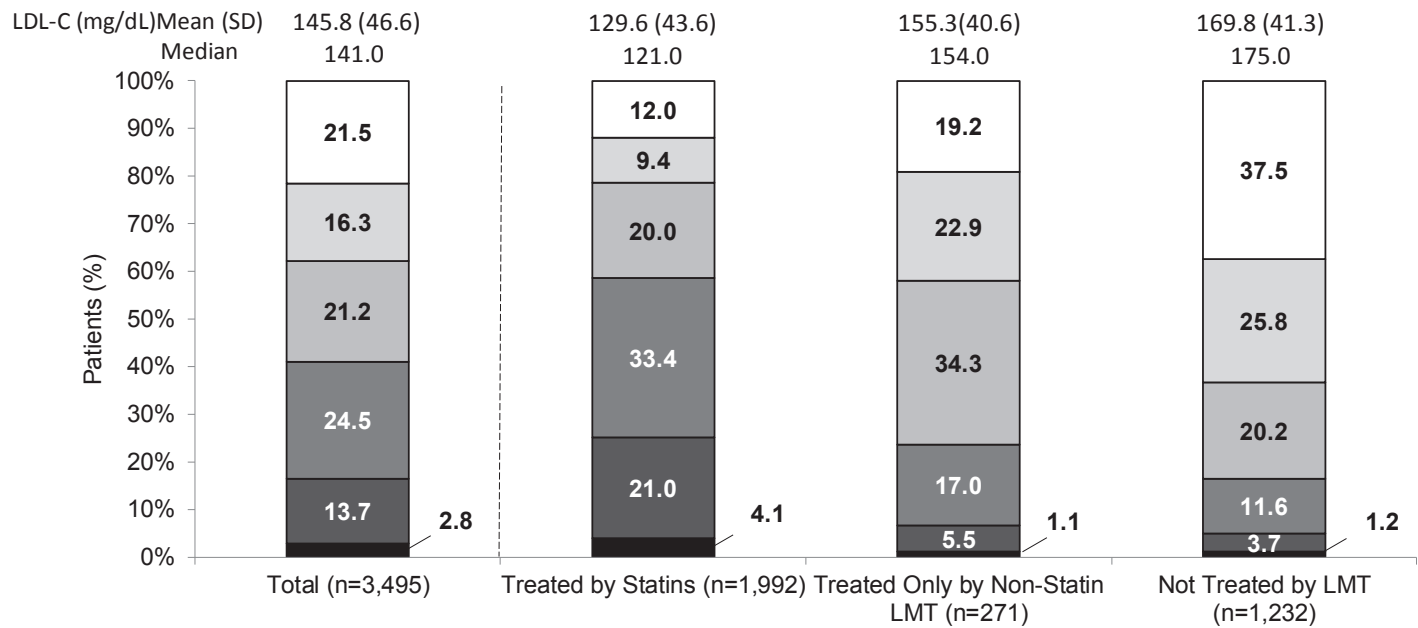

B) FH-D Patients

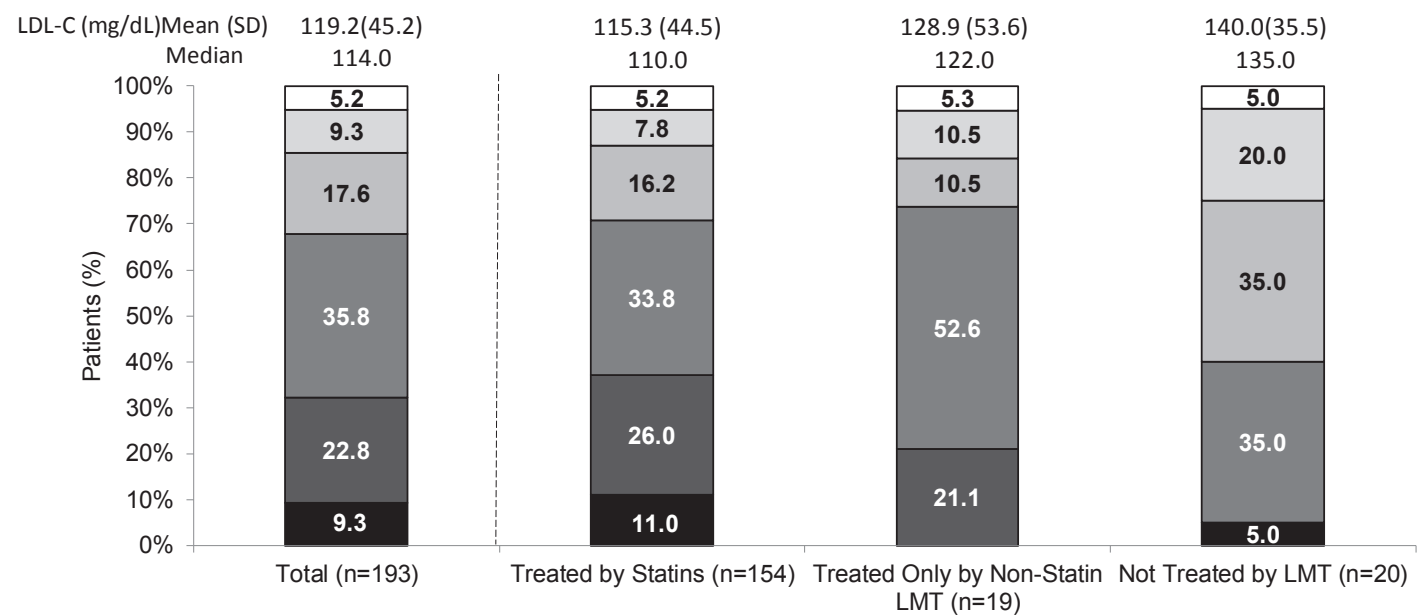

\section{C) FH-S Patients}

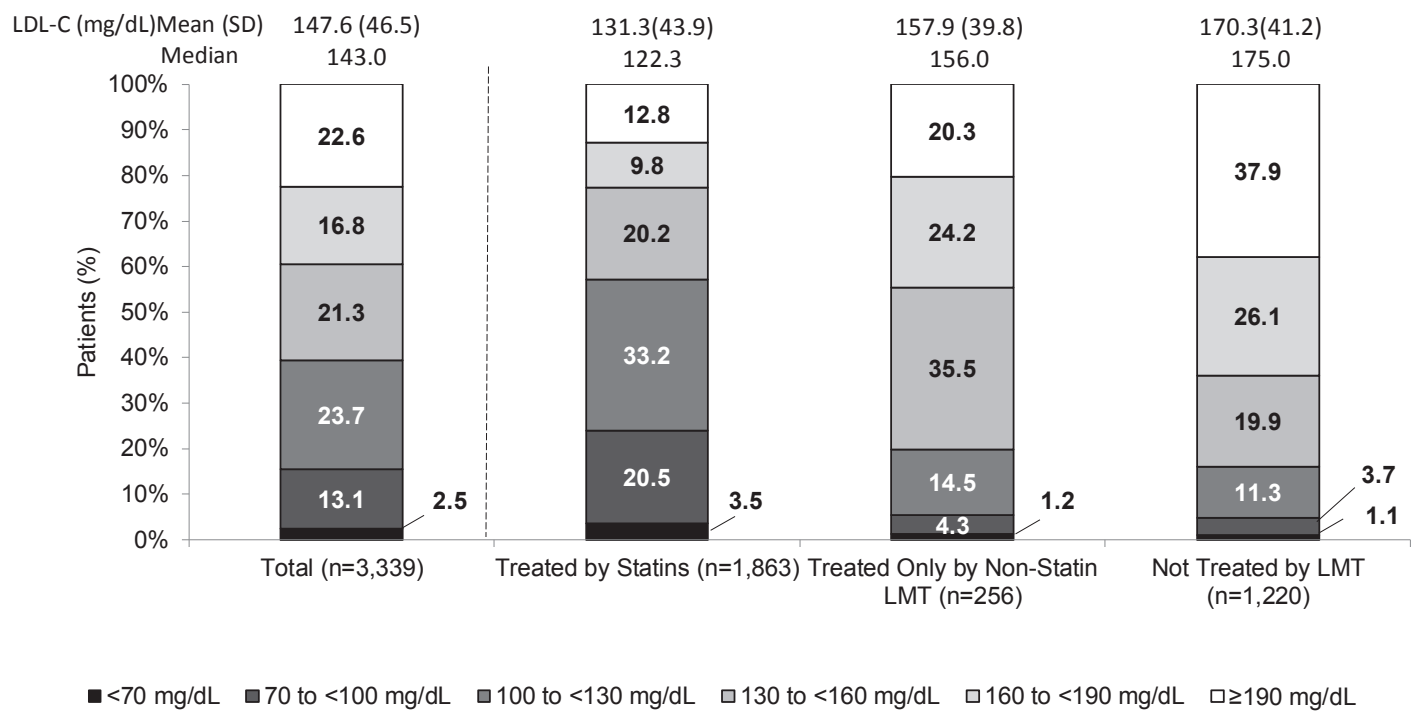

Fig. 3. LDL-C levels at index date for all patients, FH-D patients, and FH-S patients 


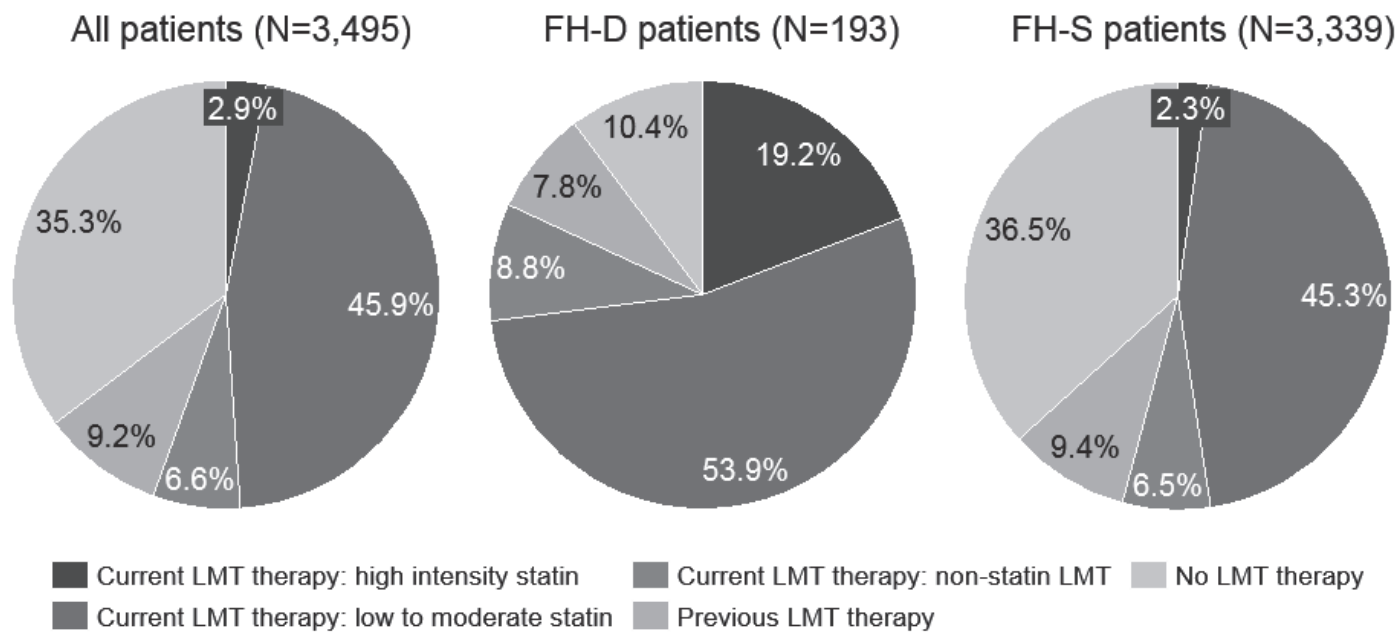

Fig. 4. LMT utilization for all patients, FH-D patients, and FH-S patients

patients, LDL-C management was inadequate regardless of the presence of CAD. In addition, few FH-D and FH-S patients with CAD achieved the LDL-C target level of $<70 \mathrm{mg} / \mathrm{dL}$ recommended by the newly revised 2017 JAS guidelines ${ }^{2)}$.

Inadequate treatment of $\mathrm{FH}$ has been reported worldwide ${ }^{16,23)}$, and the results of the present study revealed that Japan is no exception. Low-to-moderateintensity statins were found to be the most commonly prescribed LMTs (45.9\% of all patients), and 35.3\% of all patients had no record of LMT prescriptions during the study period. The majority of the patients treated with statins remained on monotherapy even when their LDL-C levels were high. The JAS guidelines recommend drug therapy for patients with $\mathrm{FH}$, using statins as first line followed by a combination therapy with non-statin LMT in further lines of therapy if monotherapy with statins was inadequate ${ }^{13)}$. While the JAS guidelines recommend aggressive statin therapy for $\mathrm{FH}$ patients, typically in the form of high doses of high-intensity statins, the majority of the patients in this study were found to receive low-tomoderate-intensity statins. Inadequate management of LDL-C levels may be partially related to lower proportions of patients receiving LMTs, especially high-intensity statins.

Low diagnostic rate of $\mathrm{FH}$ is another crucial factor for the lack of optimal therapy for high LDL-C levels. In this study, $5.5 \%$ of all patients had an existing diagnosis of FH. Our study also calculated the diagnosis rate among all patients currently treated with any statins and high-intensity statin to be only $8.3 \%$ and $35.9 \%$, respectively. A report from Shiba et al. suggests a high likelihood of patients with LDL-C $\geq 190 \mathrm{mg} / \mathrm{dL}$ having $\mathrm{FH}^{24)}$. We defined FH-S patients as patients with LDL-C $\geq 190 \mathrm{mg} / \mathrm{dL}$ regardless of the status of the LDL-C-lowering therapy; therefore, we would expect our study population to have a rate of diagnosis of $\mathrm{FH}$ much higher than $5.5 \%$. On the other hand, by applying a prevalence of $\mathrm{HeFH}$ in Japan of $1 / 208$, as reported in a prior study ${ }^{5}$, we could estimate that there are 372 patients with FH within our study population aged $\geq 20$ years with 2 -year baseline data $(N=77,415)$. We can then calculate that our study population has a $51.9 \%$ diagnosis rate $(193 \mathrm{FH}-\mathrm{D}$ patients out of 372). However, this value needs to be interpreted with caution due to selection bias (e.g., exclusion of patients without LDL-C $\geq 190 \mathrm{mg} / \mathrm{dL}$ and larger acute-care hospital as a data source), making the diagnostic rate of $51.9 \%$ an upper limit to an overestimation of the true diagnosis rate. Nevertheless, given the aforementioned, we could conclude that the diagnostic rate of $\mathrm{FH}$ in facilities associated with acute-care hospitals is significantly higher than that previously reported for the general population, which likely represents higher prevalence and higher awareness of $\mathrm{FH}$ in this clinical setting.

The current study has also found a tendency for increased use of high-intensity statins within FH-D patients compared to all patients $(19.2 \%$ of FH-D patients vs. $2.9 \%$ of all patients). There is also a higher rate of FH-D patients as LMT therapy intensifies, highlighting the potential positive impact of an appropriate diagnosis on improving management of LDL-C levels among patients with FH. In fact, the average LDL-C levels reported in our study were considerably lower for FH-D patients $(119.2 \mathrm{mg} / \mathrm{dL})$ than for FH-S patients $(147.6 \mathrm{mg} / \mathrm{dL})$. This may reflect the trend of FH-D patients to seek treatment following a formal diagnosis and physicians to appropriately pursue more 
aggressive LDL-C-lowering therapy. It is important for Japanese clinicians to be acutely suspicious and proactively aware of the following diagnostic criteria for adult $\mathrm{FH}$ patients (meeting at least 2 of the following 3 items): 1) hyper-LDL cholesterolemia (LDL-C levels before treatment: $\geq 180 \mathrm{mg} / \mathrm{dL}$ ); 2) tendon xanthoma (tendon xanthoma on the dorsal hands, elbows, and knees, or Achilles tendon thickening of $\geq 9 \mathrm{~mm}$ on radiography) or nodular xanthoma on the skin; and 3) family history of $\mathrm{FH}$ within the second-degree relatives or premature CAD. HoFH can also be diagnosed based on clinical features: serum total cholesterol of $\geq 600$ $\mathrm{mg} / \mathrm{dL}$, cutaneous xanthoma, premature CAD during childhood, and parents' family history of $\mathrm{HeFH}^{13)}$.

There are several limitations in this study. First, the use of a hospital database may represent selection bias that limits the generalizability of the results to the overall and FH populations in Japan. The data collected were limited to reflect clinical practice at larger acute-care hospital settings and patients who returned to the same hospital or clinic for continuous care with multiple LDL-C levels measured, limiting the generalizability of the results to continuously followed patients at larger hospitals. Secondly, it was not possible to follow patients who went to a different hospital or clinic, or to identify the same patient receiving care at more than one hospital in this database. The database also does not capture health services and prescriptions outside of the hospital. Third, patient demographics and clinical characteristics were limited to the information available during the 2-year pre-index period, which may have restricted the information used for clinical decision regarding the types of LMT prescribed or the diagnosis of $\mathrm{FH}$ itself. On the other hand, some of the patients who would have had pre-treated LDL-C $\geq 190$ $\mathrm{mg} / \mathrm{dL}$ prior to the 2-year pre-index period were not included in the current analysis. Fourth, the definition of FH-D and FH-S does not perfectly represent the diagnosis of $\mathrm{FH}$ used in clinical practice. We used LDL-C levels only to define FH-S populations without considering other factors such as family history, xanthoma, or possible secondary cause of hyperlipidemia. Furthermore, we could not exclude patients with a recorded diagnosis of $\mathrm{FH}$ in order to obtain insurance approval for certain prescriptions such atorvastatin $40 \mathrm{mg}$, which is only approved for patients with $\mathrm{FH}$ in Japan. Our study also used a relatively strict definition of "current therapy," defined by a recorded prescription on or within 30 days of the index date. In addition, the higher rate of females as well as younger patients included may be the reason for the lower intensity of LMT in the current analysis. Lastly, the current database did not allow an assessment of whether $\mathrm{FH}$ patients had lowered their LDL-C levels by more than
$50 \%$ in this study. This may partly explain our findings showing that some of the treated patients remained above the LDL-C level of $100 \mathrm{mg} / \mathrm{dL}$.

\section{Conclusion}

The results of the present study suggest that treatment and management of LDL-C levels among patients with diagnosed and suspected FH in Japan remain suboptimal. The results also revealed a low rate of FH diagnosis in real-world clinical routine practice in Japan. There is an urgent need for more proactive diagnosis of $\mathrm{FH}$ and aggressive treatment targeting LDL-C levels $<100 \mathrm{mg} / \mathrm{dL}$ for patients with FH in Japan.

\section{Acknowledgments and Notice of Grant Support}

This study was funded by Sanofi and Regeneron Pharmaceuticals, Inc.. We thank Carol Hudson and Robert Sanchez from Regeneron Pharmaceuticals, Inc. for their contributions to critical review of the manuscript. Editorial assistance was provided by Ayumi Tomari from IMS Japan.

\section{Conflict of Interest}

This study was funded by Sanofi and Regeneron Pharmaceuticals, Inc.. Dr. Teramoto received honoraria and scholarship grants from Sanofi, Daiichi-Sankyo, Bayer, Takeda Pharmaceutical, Astellas Pharma, Amgen Astellas BioPharma, Pfizer, Shionogi, Eli Lilly, Kissei Pharmaceutical, Mochida Pharmaceutical, MSD and Kowa; and was endowed courses at his affiliation by Bayer, Shionogi, Mochida Pharmaceutical, MSD, Aska Pharmaceutical and Kowa. Dr. Arai received honoraria from Sanofi, MSD, Daiichi-Sankyo, Astellas Pharma, Amgen Astellas BioPharma and Kowa. Dr. Yamashita received honoraria and scholarship grants from Sanofi, Kowa, Otsuka Pharmaceutical, Shionogi, Bayer, MSD, Takeda Pharmaceutical, Sanwa Kagaku Kenkyusho, Ono Pharmaceutical, Astellas Pharma, Daiichi-Sankyo, Astra Zeneca, Medical Review, Skylight Biotech, Kaken Pharmaceutical, Pfizer, Bristol-Meyers Squibb, Amgen Astellas BioPharma, Aegerion Pharmaceuticals, Toa Eiyo, Nippon Boehringer Ingelheim, National lnstitute of Biomedical lnnovation, Kyowa Medex, Mochida Pharmaceutical, Hayashibara, Teijin Pharma and Kissei Pharmaceutical. Dr. Ozaki and Mr. Kai are employees of Sanofi, and Mr. Kai have some shares in Sanofi, during the conduct of this study. Mr. Crawford was an employee of IMS Japan, a healthcare consulting firm contracted for this research, during the conduct of this study. 


\section{References}

1) Teramoto T, Sasaki J, Ishibashi $S$, Birou $S$, Daida H, Dohi S, Egusa G, Hiro T, Hirobe K, Iida M, Kihara S, Kinoshita M, Maruyama C, Ohta T, Okamura T, Yamashita S, Yokode M, Yokote K, Harada-Shiba M, Arai H, Bujo H, Nohara A, Ohta T, Oikawa S, Okada T, Wakatsuki A. Familial Hypercholesterolemia. J Atheroscler Thromb. 2014; 21: 6-10

2) Japan Atheroscoerlsis Society. Japan Atheroscoerlsis Society (JAS) Guidelines for Prevention of Atherosclerotic Cardiovascular Diseases 2017. (in Japanese)

3) Harada $T$, Inagaki-Tanimura $K$, Nagao $M$, Sato $Y$, Sudo M, Okajima F, Sugihara H, Oikawa S. Frequency of Achilles Tendon Xanthoma in Patients with Acute Coronary Syndrome. J Atheroscler Thromb. 2017; 24: 949-953

4) Cuchel M, Bruckert E, Ginsberg HN, Raal FJ, Santos RD, Hegele RA, Kuivenhoven JA, Nordestgaard BG, Descamps OS, Steinhagen-Thiessen E, Tybjærg-Hansen A, Watts GF, Averna M, Boileau C, Borén J, Catapano AL, Defesche JC, Hovingh GK, Humphries SE, Kovanen PT, Masana L, Pajukanta P, Parhofer KG, Ray KK, Stalenhoef AF, Stroes E, Taskinen MR, Wiegman A, Wiklund O, Chapman MJ; European Atherosclerosis Society Consensus Panel on Familial Hypercholesterolaemia. Homozygous familial hypercholesterolaemia: new insights and guidance for clinicians to improve detection and clinical management. A position paper from the Consensus Panel on Familial Hypercholesterolaemia of the European Atherosclerosis Society. Eur Heart J. 2014; 35: 2146-2157

5) Mabuchi H, Nohara A, Noguchi T, Kobayashi J, Kawashiri MA, Tada H, Nakanishi C, Mori M, Yamagishi M, Inazu A, Koizumi J; Hokuriku FH Study Group. Molecular genetic epidemiology of homozygous familial hypercholesterolemia in the Hokuriku district of Japan. Atherosclerosis. 2011; 214: 404-407

6) Pang J, Lansberg PJ, Watts GF. International Developments in the Care of Familial Hypercholesterolemia: Where Now and Where to Next? J Atheroscler Thromb. 2016; 23: 505-519

7) Mabuchi H. Half a Century Tales of Familial Hypercholesterolemia (FH) in Japan. J Atheroscler Thromb. 2017; 24: 189-207

8) Robinson JG. Management of familial hypercholesterolemia: a review of the recommendations from the National Lipid Association Expert Panel on Familial Hypercholesterolemia. Journal of managed care pharmacy: J Manag Care Pharm. 2013; 19: 139-149

9) Hopkins PN, Toth PP, Ballantyne CM, Rader DJ; National Lipid Association Expert Panel on Familial Hypercholesterolemia. Familial hypercholesterolemias: prevalence, genetics, diagnosis and screening recommendations from the National Lipid Association Expert Panel on Familial Hypercholesterolemia. J Clin Lipidol. 2011; 5: S9-17

10) Jansen AC, van Aalst-Cohen ES, Tanck MW, Trip MD, Lansberg PJ, Liem AH, van Lennep HW, Sijbrands EJ, Kastelein JJ. The contribution of classical risk factors to cardiovascular disease in familial hypercholesterolaemia: data in 2400 patients. J Intern Med. 2004; 256: 482-490

11) Alonso R, Mata N, Castillo S, Fuentes F, Saenz P, Muñiz O, Galiana J, Figueras R, Diaz JL, Gomez-Enterría P,
Mauri M, Piedecausa M, Irigoyen L, Aguado R, Mata P; Spanish Familial Hypercholesterolaemia Group. Cardiovascular disease in familial hypercholesterolaemia: influence of low-density lipoprotein receptor mutation type and classic risk factors. Atherosclerosis. 2008; 200: 315-321

12) Mabuchi H, Koizumi J, Shimizu M, Takeda R. Development of coronary heart disease in familial hypercholesterolemia. Circulation. 1989; 79: 225-232

13) Harada-Shiba $M$, Arai H, Oikawa $S$, Ohta T, Okada $T$, Okamura T, Nohara A, Bujo H, Yokote K, Wakatsuki A, Ishibashi S, Yamashita S. Guidelines for the management of familial hypercholesterolemia. J Atheroscler Thromb. 2012; 19: 1043-1060

14) European Association for Cardiovascular Prevention \& Rehabilitation, Reiner Z, Catapano AL, De Backer G, Graham I, Taskinen MR, Wiklund O, Agewall S, Alegria E, Chapman MJ, Durrington P, Erdine S, Halcox J, Hobbs R, Kjekshus J, Filardi PP, Riccardi G, Storey RF, Wood D; ESC Committee for Practice Guidelines (CPG) 20082010 and 2010-2012 Committees. ESC/EAS Guidelines for the management of dyslipidaemias: the Task Force for the management of dyslipidaemias of the European Society of Cardiology (ESC) and the European Atherosclerosis Society (EAS). Eur Heart J. 2011; 32: 1769-1818

15) Catapano AL, Graham I, De Backer G, Wiklund O, Chapman MJ, Drexel H, Hoes AW, Jennings CS, Landmesser U, Pedersen TR, Reiner Ž, Riccardi G, Taskinen MR, Tokgozoglu L, Verschuren WM, Vlachopoulos C, Wood DA, Zamorano JL; Authors/Task Force Members; Additional Contributor. 2016 ESC/EAS Guidelines for the Management of Dyslipidaemias. Eur Heart J. 2016; 37: 2999-3058

16) Nordestgaard BG, Chapman MJ, Humphries SE, Ginsberg HN, Masana L, Descamps OS, Wiklund O, Hegele RA, Raal FJ, Defesche JC, Wiegman A, Santos RD, Watts GF, Parhofer KG, Hovingh GK, Kovanen PT, Boileau C, Averna M, Borén J, Bruckert E, Catapano AL, Kuivenhoven JA, Pajukanta P, Ray K, Stalenhoef AF, Stroes E, Taskinen MR, Tybjærg-Hansen A; European Atherosclerosis Society Consensus Panel. Familial hypercholesterolaemia is underdiagnosed and undertreated in the general population: guidance for clinicians to prevent coronary heart disease: consensus statement of the European Atherosclerosis Society. Eur Heart J. 2013; 34: 3478-3490a

17) Tasaki H, Miyamoto M, Kubara T, Kamezaki F, Tanaka S, Yamashita K, Tsutsui M, Nakashima Y. Cross-Over Trial of Intensive Monotherapy With Atorvastatin and Combined Therapy With Atorvastatin and Colestimide for Japanese Familial Hypercholesterolemia. Circ J. 2005; 70: $14-20$

18) Harada-Shiba M, Sugisawa T, Makino H, Abe M, Tsushima M, Yoshimasa Y, Yamashita T, Miyamoto Y, Yamamoto A, Tomoike H, Yokoyama S. Impact of statin treatment on the clinical fate of heterozygous familial hypercholesterolemia. J Atheroscler Thromb. 2010; 17: 667-674

19) Teramoto $T$, Kashiwagi A, Mabuchi H, Investigators JL. Status of lipid-lowering therapy prescribedbased on recommendations in the 2002 report of the Japan Atherosclerosis Society Guideline for Diagnosis and Treatment of Hyperlipidemia in Japanese Adults: A study of the Japan Lipid Assessment Program (J-LAP). Curr Ther Res 
Clin Exp. 2005; 66: 80-95

20) Teramoto T. Special Drug Use-Results Survey of Lipitor Tablets (ALWAYS): Interim Report. Therapeutic Research. 2011; 32: 1587-1603

21) Stone NJ, Robinson JG, Lichtenstein AH, Bairey Merz CN, Blum CB, Eckel RH, Goldberg AC, Gordon D, Levy D, Lloyd-Jones DM, McBride P, Schwartz JS, Shero ST, Smith SC Jr, Watson K, Wilson PW; American College of Cardiology/American Heart Association Task Force on Practice Guidelines. 2013 ACC/AHA guideline on the treatment of blood cholesterol to reduce atherosclerotic cardiovascular risk in adults: a report of the American College of Cardiology/American Heart Association Task Force on Practice Guidelines. J Am Coll Cardiol. 2014; 63: 2889-2934

22) Bujo H, Takahashi K, Saito Y, Maruyama T, Yamashita $S$, Matsuzawa Y, Ishibashi S, Shionoiri F, Yamada N, Kita T;
Research Committeon Primary Hyperlipidemia of the Ministry of Health, Labour, and Welfare of Japan. Clinical features of familial hypercholesterolemia in Japan in a database from 1996-1998 by the research committee of the ministry of health, labour and welfare of Japan. J Atheroscler Thromb. 2004; 11: 146-151

23) Kuiper JG, Sanchez RJ, Houben E, Heintjes EM, Penning-van Beest FJA, Khan I, van Riemsdijk M, Herings RMC. Use of Lipid-modifying Therapy and LDL-C Goal Attainment in a High-Cardiovascular-Risk Population in the Netherlands. Clin Ther. 2017; 39: 819-827 e811

24) Harada-Shiba M, Arai H, Okamura T, Yokote K, Oikawa S, Nohara A, Okada T, Ohta T, Bujo H, Watanabe M, Wakatsuki A, Yamashita S. Multicenter study to determine the diagnosis criteria of heterozygous familial hypercholesterolemia in Japan. J Atheroscler Thromb. 2012; 19: 1019-1026 


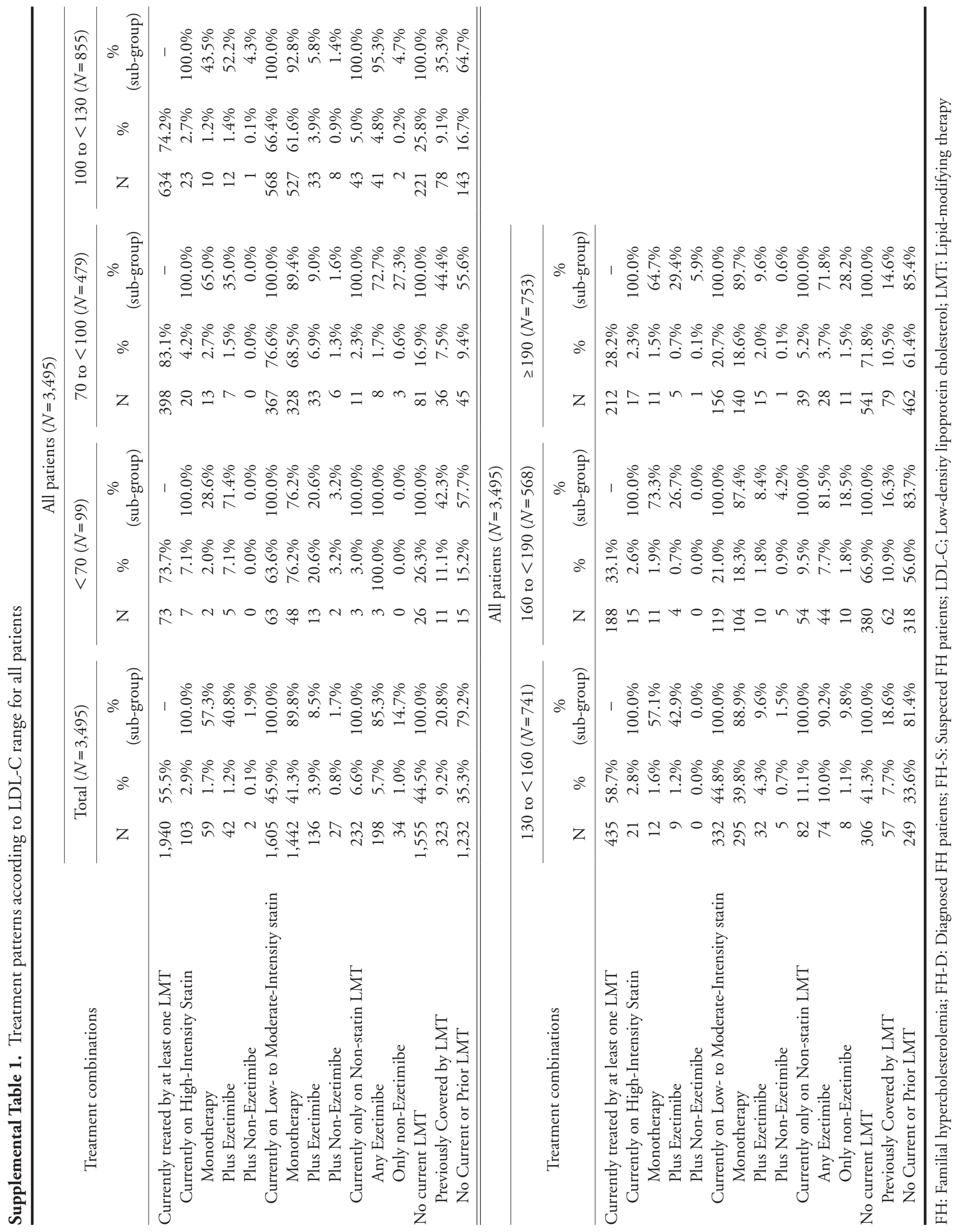




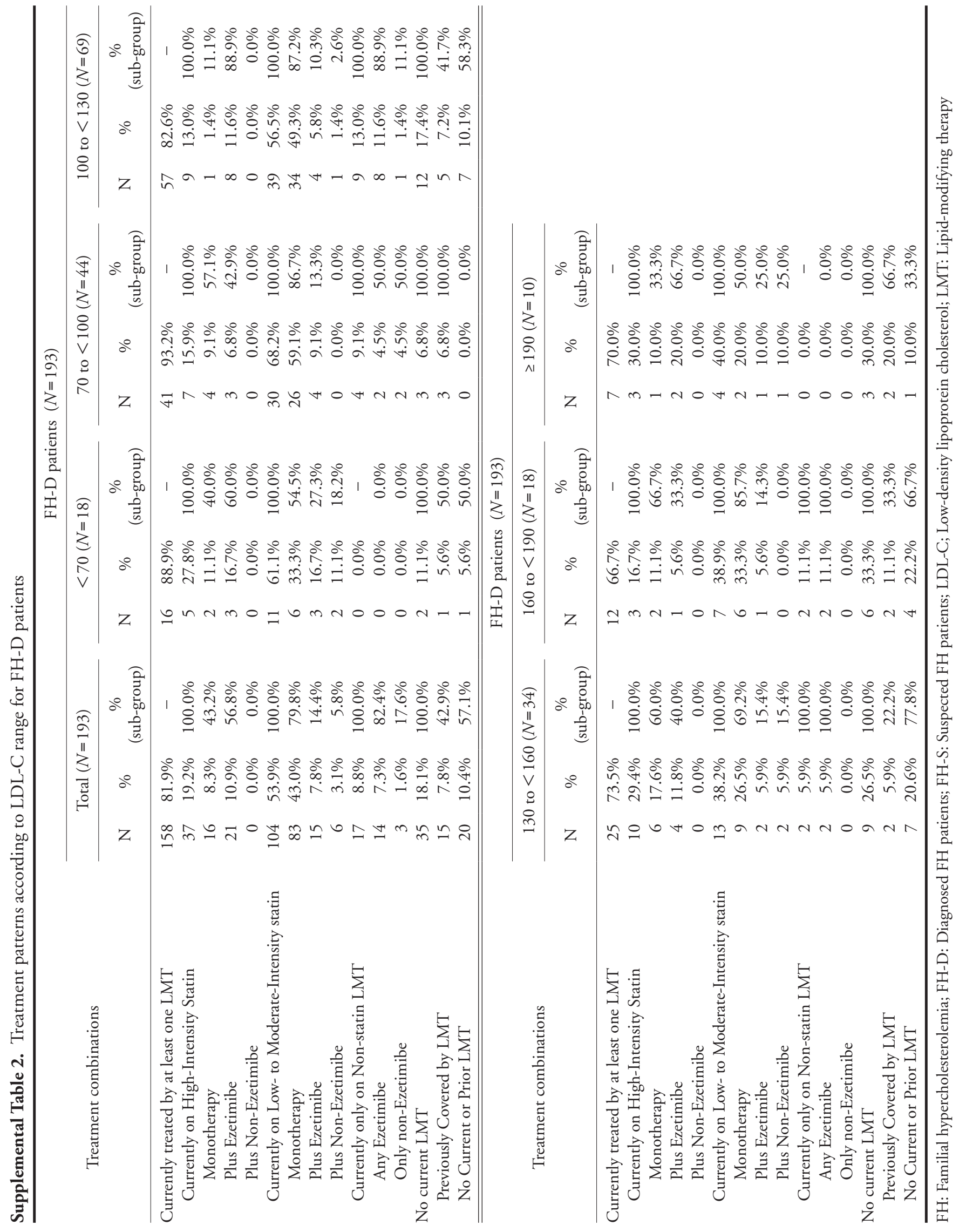




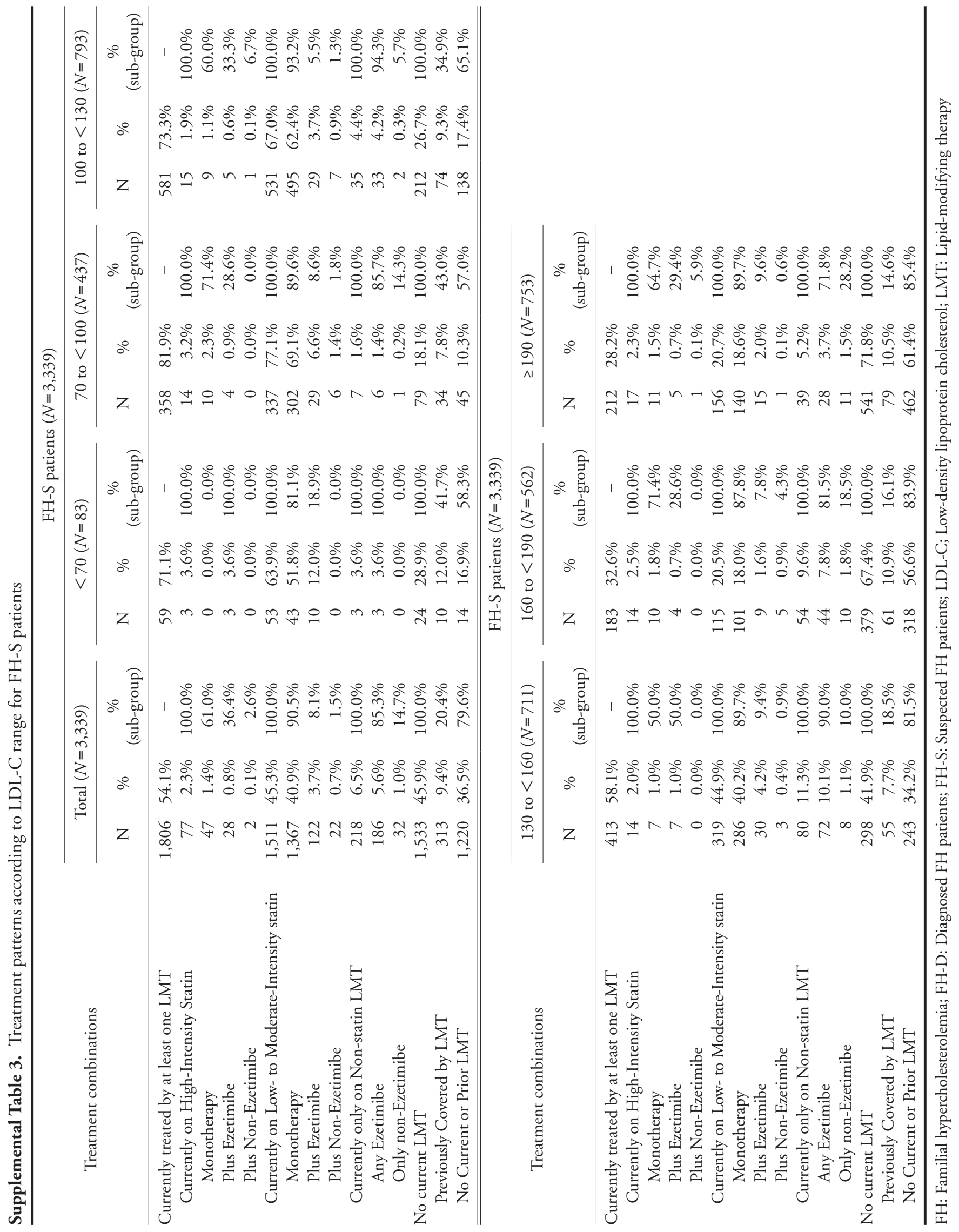

